\title{
LESSONS IN HAND WASHING: INFLUENCE OF OCCUPATION, HABIT AND SANITATION ON THE TYPE AND BURDEN OF HAND MICROFLORA
}

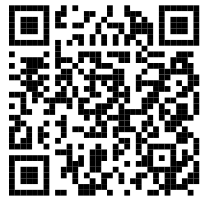

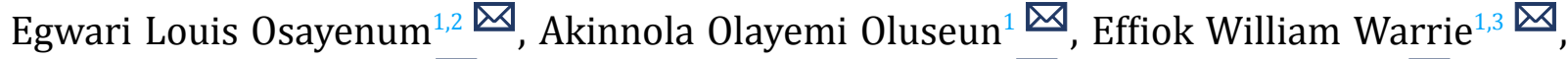 \\ Kilani Adetunji Musbau ${ }^{4}$, Uzowuihe Xavier Chukwuma ${ }^{1}$ and Adegbayi Tolu ${ }^{1} \mathbb{B}$ (D) \\ ${ }^{1}$ Department of Biological Sciences, Covenant University, Ota, Ogun, Nigeria \\ ${ }^{2}$ QSM Training and Consulting Limited, 68 Randle Avenue, Surulere, Lagos, Nigeria \\ ${ }^{3}$ National Agency for Food, Drug Administration and Control, Lagos, Oshodi, Nigeria \\ ${ }^{4}$ Department of Microbiology, Federal University Dutsinma, Katsina State, Nigeria
}

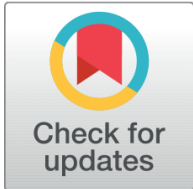

Received 29 May 2021

Accepted 9 June 2021

Published 30 June 2021

Corresponding Author

Egwari Louis Osayenum, louis.egw

ari@covenantuniversity.edu.ng

DOI $10.29121 /$

granthaalayah.v9.i6.2021.3976

Funding: This research received no specific grant from any funding agency in the public, commercial, or not-for-profit sectors.

Copyright: (C) 2021 The Author(s). This is an open access article distributed under the terms of the Creative Commons Attribution License, which permits unrestricted use, distribution, and reproduction in any medium, provided the original author and source are credited.

\section{ABSTRACT}

The hand is an important vehicle in disease transmission both in the healthcare setting and in communities. This study accessed the level of compliance to hand hygiene requirements among different professionals within and outside the hospital setting.in addition to the role of good hand hygiene practice in reducing the microbial population of hands. Structured questionnaire to access compliance by the various study group was analyzed statistically. Swabs of hands and contact surfaces were collected before and after different treatment application ranging from washing with soap, use of sanitizers or a combination of treatment and assessed microbiologically. Knowledge of the importance of hand hygiene did not translate to better hand hygiene practice among health workers compared to groups outside the health profession. There was a higher preference for water for anal cleansing after defecation as opposed to the use of tissue paper by male than female $(\mathrm{p}<0.05)$. The bacterial load in unwashed hands ranged from $10^{6} \mathrm{CFU} / \mathrm{ml}$ for Proteus and Streptococcus species to $10^{10} \mathrm{CFU} / \mathrm{ml}$ for Klebsiella and Staphylococcus species. Hand washing without application of sanitizer resulted in 1-2 $\log _{10}$ CFU reduction depending on bacterial species ( $p>0.05$ ). Application of sanitizer to unwashed hands resulted in 2-7 $\log _{10} \mathrm{CFU}$ reduction for most bacterial species $(\mathrm{p}<0.05)$. Hands were re-colonized with same flora within one week of decolonizing. The multiple factors associated with re-colonization of decolonized hands pose the question as to how frequent should hands be washed?

Keywords: Microorganisms, Hand hygiene, Sanitizer, Occupation, Habit, Microbial load 


\section{INTRODUCTION}

The World Health Organization approved the five Moments of hand hygiene in healthcare practice to significantly prevent transmission of pathogens from healthcare providers to patients. The success of this scheme is dependent on compliance and availability of structure for effective deployment of hand hygiene guidelines. Prevention and management of infection is the responsibility of all staff working in health and social care, and an integral element of patient safety programs "Essential Practice for Infection Prevention and Control: Guidance for Nursing Staff" (2014). Preventing infections requires sustained compliance with a number of good practice areas - including the provision of clean environment, aseptic techniques, and the management of invasive devices. However, evidence shows that improving hand hygiene contributes significantly to the reduction of healthcare associated infections (HCAIs) Pratt et al. (2007). Hands are therefore a very efficient vehicle for transferring microorganisms.

The plague of Ebola that ravaged many West African countries including Nigeria was a case in hand that calls for prompt epidemiological intervention in outbreaks. The rapid spread of the disease and the attendant casualty was associated with inadequate epidemiological structure to curb the spread in the most hit countries. It is however interesting to note that Ebola has existed ever since the early 1970s when the first outbreaks were reported in southern Sudan in 1976 (284 cases and 117 deaths and in the Democratic Republic of Congo (318 cases and 280 deaths) "World Health Organization. Ebola Haemorrhagic Fever in Sudan" (1976); "World Health Organization. Ebola Haemorrhagic Fever in Zaire" (1976). Between 2000 and 2001 another outbreak occurred in Uganda with 425 cases and 224 deaths Francesconi et al. (2003). All these evidently were cases of contacts with infected animals, humans and carcasses depicting laxity in institution of epidemiological structures. The 2014 re-emergence of Ebola in West Africa and its introduction into America and Europe calls for concerted efforts to establish epidemiological structures and institute frequent surveillance for early detection and control of potential outbreaks. Now the world is confronted with another deadly outbreak of measles and even in adults. These are pointers to stricter epidemiological surveillance and control measures to be put in place to forestall unforeseen incidences that will be both cost intensive to control and severe in morbidity and mortality especially in low and medium income countries where the effects of such outbreaks are usually devastating.

The $15^{\text {th }}$ of October 2014 was observed as hand washing day globally. For us in Nigeria and Africa this has special significance considering the recurring scourge of Ebola. Though Nigeria successfully contained the entrance of the virus and prevented spread to the larger community, major lessons in epidemiology were learnt. Continued surveillance became a necessity to control and prevention and we have joined the global health community annually in observing Clean Hand as a means of creating awareness on the importance of clean hands in the control and spread of diseases both in the hospital environment and the general community. In essence it 
is paramount to establish and assess compliance to guidelines in our community.

Infection or disease may be caused by different groups of microorganisms such as bacteria, fungi, viruses or prions and can result in a wide variety of infections that include, for example, urinary tract, wound, respiratory, blood, and bone and skin infections British Association for Parenteral and Enteral Nutrition (2008); "The Cost of Disease Related Malnutrition in the UK and Economic Considerations for the use of Oral Nutritional Supplements (ONS) in Adults" (2005); Department and Health (2009); "Nutrition Screening Survey in the UK in 2007: A Report by BAPEN. Nutrition Screen Survey and Audit of Adults on Admission to Hospital, Care Homes and Mental Health Units" (2008). Health and social care settings can provide a challenging environment in which to manage risks associated with the transfer of microorganisms from patient to patient or between the environment, equipment, staff and patients. Understanding how infections occur and how different microorganisms act and spread is critical to preventing infections Currie et al. (2011).

Hospitals by design provide peculiar settings for transmission of microorganisms with ease compared with normal home or other non-hospital environments. Additionally, patients may have direct contact with a large number of people as a result of their 24 hours a day care needs, and this allows for many more opportunities for transfer of microorganisms, some of which may be resistant to antibiotics. It is important to recognize that the hands of healthcare staff will always carry bacteria, be it their own bacteria or those that have attached as a result of activities (handling equipment, touching surfaces or patients). Standard infection control precautions, formerly known as universal precautions, underpin routine safe practice, protecting both staff and clients from microorganisms that may cause infection. Similarly, adequate community hygiene is also necessary in controlling spread of infections that originate from the community. Since hand is a major vessel for transmission of pathogens, hand hygiene and how it helps in infection control and prevention is the objective of this study. The present study is therefore designed to develop epidemiological strategies for continuous surveillance, monitoring and application of epidemiological data for prevention and intervention in the course of an outbreak focusing our attention on the health sector and the general community as represented by the university community and food vending establishment.

\section{MATERIALS AND METHODS}

The study was based on responses to a structured questionnaire (Tables 1,2 and 3 ) followed by laboratory investigation. Centers used for the study include a private university, hospitals (university hospital- UH, and specialist hospital- $\mathrm{SH}$ ), medical laboratories (hospital laboratories- SHL and private diagnostic laboratories-PDL) and food vending outlets. On privacy and ethical considerations, the full identity of the study centers was not disclosed. Questionnaires were distributed to participants after a session of briefing and only those that signed a consent form to abide with 
the conditions of the study were admitted. Questionnaires were filled and retrieved same day and sorted out for further study. Based on response to the questions samples were collected for microbiological investigations. In addition to sampling of hands which included the palm, dorsum, finger cleft and under finger nails, inanimate objects such as door handles of major entrances to the Centers and toilets were sampled.

Sampling was categorized as follows; wet unwashed hands, hands washed with liquid soap, hand sanitized with alcohol based hand robs and hand washed and sanitized. Samples were collected from other groups of volunteers who used the toilet and cleaned the anus either with water or used tissue paper. This was done to assess the contributory role of toilet hygiene habit on the microbial load and diversity on the hands. Samples were collected with moistened swabs (sterile albumin coated swab; Oxoid, and moistened with sterilized distilled water) before and after each hand treatment, and then cultured for both qualitative and quantitative microbial assessment. A selected group was followed up to determine the effect of improving toilet hygiene on the microbial load and type on hands. This group consisting of 20 subjects was given hand sanitizers and to use each time they visited the toilet, handled clinical specimens or came in contact with body fluid. They were also instructed to adhere strictly to the 5 Moments of hand hygiene. In order to identify sources and causes for re-colonization of hands in subjects practicing good hand hygiene, swabs from door handles of classrooms, offices, laboratories and toilets were cultured before and after disinfection with $70 \%$ methanol. The 20 subjects were then assigned to make 1-minute hand contact with the door handles; the left to un-disinfected handles and right to disinfected. The hands were then swabbed and cultured.

Microbiological investigations included culturing for bacteria and fungi. Media used were Nutrient agar, MacConkey agar, Deoxycholate Citrate agar, Eosin Methylene blue agar, Simmon Citrate agar, Baird Parker medium, Blood agar plates, Mannitol Salt agar, Potato Dextrose agar and Malt Extract agar. The isolates were identified by microscopy, cultural features and biochemical tests.

\section{Statistical analysis}

Data on microbial population for each parameter studied was log-transformed and analyzed by simple linear regression using 95\% confidence intervals. The results were confirmed using Kruskal-Wallis analysis appropriate for non-normally distributed data. Analyses were undertaken using the IBM SPSS Statistics version 24 software packages and MS Excel. For normally distributed data, t-tests were used to compare two treatments. Were three parameters are considered together, One-way ANOVA was performed with the Kukey's HSD or Dunnett's as post hoc tests depending on the homogeneity of the variance. 


\section{RESULTS}

$\mathrm{s}$ an overview of the knowledge and corresponding attitudinal response of classified groups of students and staff in a university on requirements for clean hands. While it was obvious that the groups that handle potentially hazardous infectious materials are aware of the dos and don'ts of their profession, their compliance was inconsistent with their well-informed position as evident in the no significant difference in attitude when compared with the groups with no regulations guiding their behavior with respect to keeping of nails and maintenance of proper toilet hygiene ( $p>0.05)$. The students were more nonchalant with health tips as very few keep and use sanitizers even when it was generally available for use. But the general trend with no usage of sanitizers by respondents is that it was not provided by the institution and that also include sanitary towels. Surprisingly, hand hygiene and how the 5 Moments of hand hygiene is applied was not known to all respondents despite the annual hand hygiene day observed in the institution. There is a higher preference for water for anal cleansing after defecation as opposed to the use of tissue paper by male than female $(\mathrm{p}<0.05)$. The trend described above was repeated in hospital and medical diagnostic laboratories personnel. Provision of sanitary materials was more pronounced in the laboratory section as opposed to other sectors of the hospital and correspondingly lesser compliance to hand hygiene rules by the latter (Table 2 ). Table 3 shows a more knowledge based response by food handlers as indicated by the proportion that keep nails short, provision of sanitizers and other sanitary materials and awareness and practice of food handlers' tests. The response across professional groups as to how frequent hands are washed varied but the cluster is 1 to 3 times especially during meal time.

The bacterial load in unwashed hands ranged from $10^{6} \mathrm{CFU} / \mathrm{ml}$ for Proteus and Streptococcus species to $10^{10} \mathrm{CFU} / \mathrm{ml}$ for Klebsiella and Staphylococcus species. Hand washing with soap only resulted in 1-2 $\log _{10}$ CFU reduction depending on bacterial species $(\mathrm{p}>0.05)$. When sanitizer was applied to unwashed hands microbial reduction was in the magnitude of 2-7 $\log _{10} \mathrm{CFU}$, with Enterococcus faecalis, Klebsiella spp., and Escherichia coli showing significant reduction $(\mathrm{p}<0.05)$. Further reductions $(\mathrm{p}<0.01)$ occurred ranging from 8-9 $\log _{10}$ CFU for Klebsiella spp., E. coli, Enterococcus faecalis and Staphylococcus spp., on the high side to 5-7 $\log _{10}$ CFU for Proteus spp., Streptococcus spp., Bacillus spp., and Enterobacter spp., on the down side when hands were sanitized after washing with soap and rinsed with clean water. By absolute elimination, Streptococcus spp., and Candida showed highest sensitivity when sanitization followed hand washing with soap, although sanitization alone was sufficient to completely eliminate Candida albicans from hands. Pseudomonas aeruginosa reduction was in order of $2 \log _{10} \mathrm{CFU}$ in sanitized hands and $6 \log _{10} \mathrm{CFU}$ in washed and sanitized hands (Table 4 ).

Microorganisms isolated from hands based on toilet habit and finger nails type are given in Table 5 . Significant difference in recovery between use of tissue paper and water as mode of anal cleansing after defecation was observed for E. coli, Enter- 
obacter spp., Proteus spp., Bacillus spp., and C. albicans. With respect to nail type, recovery of bacteria from hands with short nails was significantly different compared to recovery from hands of other nail types. Only C. albicans showed no difference in recovery from short nail and acrylic nails. Recovery rate from long nail and acrylic nails hand were similar in most cases except for Enterobacter spp., Staphylococcus spp., Streptococcus spp., and Bacillus spp., $(p<0.05)$.

ummarized the effect of disinfection on the microbial composition of inanimate objects and subsequent impact on the microbial load of decontaminated hands. Disinfection was more effective in areas least prone to contamination with microorganisms as seen in door handles from classrooms and offices. Laboratories and toilet door handles were frequently contaminated with bacteria, candida and molds. In relatively clean areas reduction was absolute in most cases especially for candida, molds and Gram positivecocci. When hand contact was made on surfaces not decontaminated, colonization rate was as high as 75-100\%, while hand contact on decontaminated surfaces only resulted in 10-15\% colonization.

Table 1 Data collation from university

\begin{tabular}{|c|c|c|c|c|c|c|c|c|}
\hline \multirow[t]{5}{*}{ Properties } & \multicolumn{8}{|c|}{ Number of Respondents } \\
\hline & \multicolumn{4}{|c|}{ Students $n=180$} & \multicolumn{4}{|c|}{ Staff $n=70$} \\
\hline & \multicolumn{2}{|c|}{ Microbiology } & \multicolumn{2}{|c|}{ Non-Microbiology } & \multicolumn{2}{|c|}{ Laboratory } & \multicolumn{2}{|c|}{ Others } \\
\hline & \multicolumn{2}{|c|}{$\mathbf{n}=\mathbf{8 0}$} & \multicolumn{2}{|c|}{$n=100$} & \multicolumn{2}{|c|}{$\mathbf{n}=\mathbf{5 0}$} & \multicolumn{2}{|c|}{$\mathbf{n}=\mathbf{2 0}$} \\
\hline & $\mathbf{M}$ & $\mathbf{F}$ & M & $\mathbf{F}$ & $\mathbf{M}$ & $\mathbf{F}$ & $\mathbf{M}$ & $\mathbf{F}$ \\
\hline \multicolumn{9}{|l|}{$\begin{array}{l}\text { Common } \\
\text { features }\end{array}$} \\
\hline a. Gender & 35 & 45 & 60 & 40 & 8 & 12 & 25 & 25 \\
\hline $\begin{array}{c}\text { b. Short } \\
\text { finger nails }\end{array}$ & 27 & 30 & 44 & 22 & 6 & 8 & 20 & 17 \\
\hline $\begin{array}{c}\text { c. Long } \\
\text { finger nails }\end{array}$ & 8 & 12 & 16 & 11 & 2 & 3 & 5 & 5 \\
\hline d. Implant & 0 & 3 & 0 & 7 & 0 & 1 & 0 & 3 \\
\hline $\begin{array}{l}\text { e. Water } \\
\text { hygiene }\end{array}$ & 17 & 15 & 32 & 14 & 6 & 7 & 15 & 8 \\
\hline $\begin{array}{l}\text { f. Tissue } \\
\text { paper }\end{array}$ & 18 & 30 & 28 & 26 & 2 & 5 & 10 & 17 \\
\hline \multicolumn{9}{|l|}{$\begin{array}{c}\text { g. } \\
\text { Frequency } \\
\text { of hand } \\
\text { hygiene:* }\end{array}$} \\
\hline (i) & 8 & 11 & 25 & 16 & 0 & 0 & 5 & 1 \\
\hline (ii) & 12 & 13 & 18 & 18 & 3 & 2 & 10 & 5 \\
\hline (iii) & 5 & 8 & 10 & 5 & 3 & 8 & 4 & 8 \\
\hline (iv) & 3 & 6 & 5 & 0 & 2 & 2 & 2 & 4 \\
\hline (v) & 7 & 7 & 2 & 1 & 0 & 0 & 4 & 7 \\
\hline \multicolumn{9}{|l|}{$\begin{array}{l}\text { h. Use of } \\
\text { sanitizer: }\end{array}$} \\
\hline $\begin{array}{l}\text { - When } \\
\text { provided }\end{array}$ & 16 & 28 & 38 & 28 & 6 & 8 & 22 & 17 \\
\hline
\end{tabular}




\begin{tabular}{|c|c|c|c|c|c|c|c|c|}
\hline \multicolumn{9}{|c|}{ Table 1 continued } \\
\hline - Regular & 9 & 11 & 5 & 10 & 2 & 4 & 3 & 6 \\
\hline - Don't use & 0 & 6 & 17 & 2 & 0 & 0 & 0 & 2 \\
\hline \multicolumn{9}{|l|}{$\begin{array}{c}\text { Unique } \\
\text { features }\end{array}$} \\
\hline \multicolumn{9}{|l|}{$\begin{array}{l}\text { a. Finger } \\
\text { nail } \\
\text { required to } \\
\text { be short at } \\
\text { workplace: }\end{array}$} \\
\hline - Yes & 35 & 45 & 0 & 0 & 4 & 8 & 0 & 0 \\
\hline$\bullet$ No & 0 & 0 & 60 & 40 & 4 & 4 & 25 & 25 \\
\hline \multicolumn{9}{|l|}{$\begin{array}{l}\text { b. Handles } \\
\text { clinical } \\
\text { specimens: }\end{array}$} \\
\hline - Yes & 10 & 7 & 8 & 5 & 4 & 8 & 5 & 15 \\
\hline$\bullet$ No & 25 & 38 & 52 & 35 & 4 & 4 & 20 & 10 \\
\hline \multicolumn{9}{|l|}{$\begin{array}{c}\text { c. Provided } \\
\text { at } \\
\text { workplace: }\end{array}$} \\
\hline $\begin{array}{c}\text { - liquid } \\
\text { soap }\end{array}$ & 35 & 45 & 35 & 10 & 4 & 8 & 0 & 0 \\
\hline $\begin{array}{l}\text { - sanitary } \\
\text { towels }\end{array}$ & 0 & 0 & 0 & 0 & 0 & 0 & 0 & 0 \\
\hline - sanitizers & 0 & 0 & 0 & 0 & 0 & 0 & 0 & 0 \\
\hline \multicolumn{9}{|l|}{$\begin{array}{c}\text { d. } \\
\text { Observes } \\
5 \mathrm{M} \text { of hand } \\
\text { hygiene: }\end{array}$} \\
\hline - Yes & 0 & 0 & 0 & 0 & 0 & 0 & 0 & 0 \\
\hline - No & 35 & 45 & 35 & 12 & 8 & 10 & 25 & 25 \\
\hline $\begin{array}{l}\text { • Don't } \\
\text { know }\end{array}$ & 0 & 0 & 0 & 0 & 0 & 0 & 0 & 0 \\
\hline \multicolumn{9}{|l|}{$\begin{array}{l}\text { e. Food } \\
\text { handlers' } \\
\text { test: }\end{array}$} \\
\hline - Yes & 0 & 0 & 0 & 0 & 0 & 0 & 0 & 0 \\
\hline • No & 35 & 45 & 60 & 40 & 8 & 12 & 25 & 25 \\
\hline
\end{tabular}

*i none except when bathing, ii 1-3 times mostly before and after meals, iii 4-6 times usually during meals and after use of the toilet, iv more than six times, v, infrequent

\section{Table 2 Data collation from hospitals and laboratories}

\begin{tabular}{|c|c|c|c|c|c|c|c|c|}
\hline \multirow[t]{4}{*}{ Propertie } & \multicolumn{8}{|c|}{ Number of Respondents } \\
\hline & \multicolumn{6}{|c|}{ Hospitals $n=110$} & \multirow{2}{*}{\multicolumn{2}{|c|}{$\begin{array}{c}\text { Med. Labs n }=19 \\
\text { PDL }\end{array}$}} \\
\hline & \multicolumn{2}{|c|}{ UH } & \multicolumn{2}{|c|}{ SH } & \multicolumn{2}{|c|}{ SHL } & & \\
\hline & $\mathbf{M}$ & $\mathbf{F}$ & M & $\mathbf{F}$ & $\mathbf{M}$ & $\mathbf{F}$ & M & $\mathbf{F}$ \\
\hline $\begin{array}{c}\text { Common } \\
\text { fea- } \\
\text { tures }\end{array}$ & & & & & & & & \\
\hline
\end{tabular}




\begin{tabular}{|c|c|c|c|c|c|c|c|c|}
\hline \multicolumn{9}{|c|}{ Table 2 continued } \\
\hline $\begin{array}{c}\text { a. } \\
\text { Gender }\end{array}$ & 5 & 10 & 30 & 20 & 21 & 24 & 7 & 12 \\
\hline $\begin{array}{l}\text { b. Short } \\
\text { finger } \\
\text { nails }\end{array}$ & 2 & 4 & 20 & 13 & 17 & 14 & 7 & 10 \\
\hline $\begin{array}{c}\text { c. Long } \\
\text { finger } \\
\text { nails }\end{array}$ & 3 & 6 & 10 & 5 & 4 & 6 & 0 & 2 \\
\hline $\begin{array}{c}\mathrm{d} . \\
\text { Implant }\end{array}$ & 0 & 0 & 0 & 2 & 0 & 4 & 0 & 0 \\
\hline $\begin{array}{l}\text { e. Water } \\
\text { hygiene }\end{array}$ & 1 & 3 & 19 & 5 & 15 & 7 & 1 & 0 \\
\hline $\begin{array}{l}\text { f. Tissue } \\
\text { paper }\end{array}$ & 4 & 7 & 11 & 15 & 6 & 17 & 6 & 12 \\
\hline $\begin{array}{l}\text { g. Fre- } \\
\text { quency } \\
\text { of hand } \\
\text { hygiene:* }\end{array}$ & & & & & & & & \\
\hline (i) & 0 & 0 & 2 & 1 & 0 & 0 & 0 & 0 \\
\hline (ii) & 0 & 1 & 8 & 6 & 3 & 2 & 0 & 0 \\
\hline (iii) & 3 & 4 & 10 & 5 & 12 & 10 & 4 & 8 \\
\hline (iv) & 2 & 5 & 5 & 7 & 5 & 12 & 3 & 4 \\
\hline (v) & 0 & 0 & 5 & 1 & 0 & 0 & 0 & 0 \\
\hline $\begin{array}{c}\text { h. Use } \\
\text { of sani- } \\
\text { tizer: }\end{array}$ & & & & & & & & \\
\hline $\begin{array}{c}\text { - When } \\
\text { pro- } \\
\text { vided }\end{array}$ & 1 & 2 & 18 & 4 & 12 & 15 & 5 & 10 \\
\hline$\stackrel{\bullet}{\text { Regular }}$ & 4 & 8 & 5 & 10 & 2 & 6 & 2 & 2 \\
\hline $\begin{array}{l}\text { - Don't } \\
\text { use }\end{array}$ & 0 & 0 & 7 & 6 & 7 & 3 & 0 & 0 \\
\hline $\begin{array}{c}\text { Unique } \\
\text { fea- } \\
\text { tures }\end{array}$ & & & & & & & & \\
\hline
\end{tabular}

a.

Finger

nails

required

to be

short at

work-

place:

\begin{tabular}{lcccccccc}
- Yes & 5 & 10 & 30 & 20 & 21 & 24 & 7 & 12 \\
$\bullet$ No & 0 & 0 & 0 & 0 & 0 & 0 & 0 & 0 \\
\hline
\end{tabular}




\begin{tabular}{|c|c|c|c|c|c|c|c|c|}
\hline \multicolumn{9}{|c|}{ Table 2 continued } \\
\hline $\begin{array}{c}\text { b. } \\
\text { Handles } \\
\text { clinical } \\
\text { speci- } \\
\text { mens: }\end{array}$ & & & & & & & & \\
\hline - Yes & 2 & 3 & 12 & 10 & 14 & 6 & 5 & 8 \\
\hline • No & 3 & 7 & 18 & 10 & 7 & 18 & 2 & 4 \\
\hline \multicolumn{9}{|l|}{$\begin{array}{c}\text { c. Pro- } \\
\text { vided at } \\
\text { work- } \\
\text { place: }\end{array}$} \\
\hline $\begin{array}{l}\text { - liquid } \\
\text { soap }\end{array}$ & 5 & 10 & 30 & 20 & 21 & 24 & 7 & 12 \\
\hline $\begin{array}{c}\bullet \\
\text { sanitary } \\
\text { towels }\end{array}$ & 0 & 0 & 0 & 0 & 0 & 0 & 3 & 3 \\
\hline $\begin{array}{l}\text { - sani- } \\
\text { tizers }\end{array}$ & 5 & 10 & 0 & 0 & 0 & 0 & 5 & 5 \\
\hline \multicolumn{9}{|l|}{$\begin{array}{l}\text { d. } \\
\text { Observe } \\
\text { the } 5 \mathrm{M} \\
\text { of hand } \\
\text { hygiene: }\end{array}$} \\
\hline - Yes & 2 & 5 & 8 & 5 & 8 & 11 & 1 & 2 \\
\hline$\bullet$ No & 3 & 2 & 15 & 10 & 6 & 10 & 3 & 5 \\
\hline $\begin{array}{l}\text { - Don't } \\
\text { know }\end{array}$ & 0 & 3 & 7 & 5 & 6 & 3 & 3 & 5 \\
\hline \multicolumn{9}{|l|}{$\begin{array}{l}\text { e. Food } \\
\text { han- } \\
\text { dlers' } \\
\text { test: }\end{array}$} \\
\hline - Yes & 0 & 0 & 0 & 0 & 0 & 0 & 3 & 3 \\
\hline - No & 5 & 10 & 30 & 20 & 21 & 24 & 4 & 9 \\
\hline
\end{tabular}

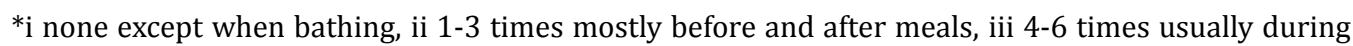
meals and after use of the toilet, iv more than six times, v, infrequent; Med. Labs, Medical Laboratories; UH, university hospital; SH, specialist hospital; SHL, specialist hospital laboratories; PDL, private diagnostic laboratories.

Table 3 Data collation from food vending outlets

\begin{tabular}{ccccc}
\hline & \multicolumn{4}{c}{ Number of Respondents } \\
& Fast Food Centre $\mathbf{n = 1 0}$ & Local Food Canteens $\mathbf{n}=\mathbf{1 3}$ \\
\hline $\begin{array}{c}\text { M } \\
\text { Common }\end{array}$ & & F & F \\
features & 4 & & 4 & 9 \\
\hline $\begin{array}{c}\text { a. Gender } \\
\text { b. Short } \\
\text { nails }\end{array}$ & 4 & 6 & 1 & 3 \\
\hline
\end{tabular}

Continued on next page 


\begin{tabular}{|c|c|c|c|c|}
\hline \multicolumn{5}{|c|}{ Table 3 continued } \\
\hline $\begin{array}{l}\text { c. Long } \\
\text { nails }\end{array}$ & 0 & 0 & 3 & 6 \\
\hline d. Implant & 0 & 0 & 0 & 0 \\
\hline $\begin{array}{l}\text { e. Water } \\
\text { hygiene }\end{array}$ & 0 & 0 & 4 & 5 \\
\hline $\begin{array}{l}\text { f. Tissue } \\
\text { paper }\end{array}$ & 4 & 6 & 0 & 4 \\
\hline \multicolumn{5}{|l|}{$\begin{array}{c}\text { g. } \\
\text { Frequency } \\
\text { of hand } \\
\text { hygiene:* }\end{array}$} \\
\hline (i) & 0 & 0 & 0 & 0 \\
\hline (ii) & 2 & 1 & 0 & 0 \\
\hline (iii) & 2 & 3 & 2 & 4 \\
\hline (iv) & 0 & 2 & 0 & 2 \\
\hline$(\mathrm{v})$ & 0 & 0 & 2 & 3 \\
\hline \multicolumn{5}{|l|}{$\begin{array}{l}\text { h. Use of } \\
\text { sanitizer: }\end{array}$} \\
\hline $\begin{array}{l}\text { - When } \\
\text { provided }\end{array}$ & 4 & 4 & 0 & 0 \\
\hline - Regular & 0 & 2 & 0 & 1 \\
\hline - Don't use & 0 & 0 & 4 & 8 \\
\hline \multicolumn{5}{|l|}{$\begin{array}{c}\text { Unique } \\
\text { features }\end{array}$} \\
\hline \multicolumn{5}{|l|}{$\begin{array}{c}\text { a. Finger } \\
\text { nail } \\
\text { required to } \\
\text { be short at } \\
\text { workplace: }\end{array}$} \\
\hline - Yes & 4 & 6 & 0 & 0 \\
\hline - No & 0 & 0 & 4 & 9 \\
\hline \multicolumn{5}{|l|}{$\begin{array}{l}\text { b. Handles } \\
\text { clinical } \\
\text { specimens: }\end{array}$} \\
\hline - Yes & 0 & 0 & 0 & 0 \\
\hline • No & 4 & 6 & 4 & 9 \\
\hline \multicolumn{5}{|l|}{$\begin{array}{c}\text { c. Provided } \\
\text { at } \\
\text { workplace: }\end{array}$} \\
\hline $\begin{array}{l}\text { - liquid } \\
\text { soap }\end{array}$ & 4 & 6 & 4 & 9 \\
\hline $\begin{array}{l}\text { - sanitary } \\
\text { towels }\end{array}$ & 4 & 6 & 0 & 0 \\
\hline - sanitizers & 4 & 6 & 0 & 0 \\
\hline \multicolumn{5}{|l|}{$\begin{array}{l}\text { d. Observe } \\
5 \mathrm{M} \text { of hand } \\
\text { hygiene: }\end{array}$} \\
\hline - Yes & 0 & 0 & 0 & 0 \\
\hline$\bullet$ No & 0 & 0 & 0 & 0 \\
\hline
\end{tabular}




\begin{tabular}{|c|c|c|c|c|}
\hline \multicolumn{5}{|c|}{ Table 3 continued } \\
\hline $\begin{array}{l}\text { - Don't } \\
\text { know }\end{array}$ & 4 & 6 & 9 & 9 \\
\hline $\begin{array}{c}\text { e. Food } \\
\text { handlers' } \\
\text { test: }\end{array}$ & & & & \\
\hline - Yes & 4 & 6 & 0 & 0 \\
\hline - No & 0 & 0 & 4 & 9 \\
\hline
\end{tabular}

*i none except whenbathing, ii 1-3 times mostly before and after meals, iii 4-6 times usuallyduring meals and after use of the toilet, iv more than six times, v, infrequent

\begin{tabular}{|c|c|c|c|c|}
\hline \multirow[t]{2}{*}{ Isolates } & & $\begin{array}{l}\text { Mean counts } \\
\text { (CFU/ml) of } \\
\text { swab samples } \\
\text { of hands }\end{array}$ & & \\
\hline & Unwashed & Washed & Sanitized & $\begin{array}{c}\text { Washed and } \\
\text { Sanitized }\end{array}$ \\
\hline $\begin{array}{l}\text { Pseudomonas } \\
\text { aeruginosa }\end{array}$ & $2.8 \times 108 \mathrm{a}$ & $6.5 \times 107 \mathrm{a}$ & 1.8 ×106 b & $3.0 \times 102 c$ \\
\hline Escherichia coli & $9.2 \times 109 a$ & $5.5 \times 107 a$ & $2.6 \times 104 b$ & $1.0 \times 101 c$ \\
\hline Klebsiella spp. & $4.0 \times 1010 \mathrm{a}$ & $7.5 \times 108 \mathrm{a}$ & $4.6 \times 105 b$ & $1.5 \times 101 \mathrm{c}$ \\
\hline $\begin{array}{l}\text { Enterobacter } \\
\text { spp. }\end{array}$ & $9.0 \times 108 \mathrm{a}$ & $5.6 \times 107 a$ & $2.0 \times 105 b$ & $7.5 \times 101 c$ \\
\hline Proteus spp. & $9.0 \times 106 \mathrm{a}$ & $2.8 \times 105 a$ & $5.0 \times 104 \mathrm{a}$ & $1.0 \times 101 \mathrm{c}$ \\
\hline $\begin{array}{l}\text { Enterococcus } \\
\text { faecalis }\end{array}$ & $2.8 \times 109 a$ & $2.5 \times 107 \mathrm{a}$ & $6.5 \times 102 c$ & $3.5 \times 101 c$ \\
\hline $\begin{array}{l}\text { Staphylococcus } \\
\text { spp. }\end{array}$ & $7.2 \times 1010 a$ & $4.5 \times 109 a$ & $3.8 \times 108 \mathrm{a}$ & $1.8 \times 102 \mathrm{c}$ \\
\hline $\begin{array}{l}\text { Streptococcus } \\
\text { spp. }\end{array}$ & $5.0 \times 106 a$ & $1.2 \times 104 \mathrm{a}$ & $2.5 \times 102 b$ & $4.0 \times 100 \mathrm{c}$ \\
\hline Bacillus spp. & $6.5 \times 107 \mathrm{a}$ & $3.0 \times 105 \mathrm{a}$ & $7.2 \times 103 \mathrm{~b}$ & $2.0 \times 101 \mathrm{c}$ \\
\hline Candida albicans & $1.0 \times 104 \mathrm{a}$ & $5.0 \times 102 \mathrm{a}$ & $0 \mathrm{c}$ & $0 \mathrm{c}$ \\
\hline
\end{tabular}

Superscriptof the same letter (a) along the same row indicates no significance at $p>0.05$; letters $b$ and $\mathrm{c}$ on the same row indicate significant microbialreduction at $\mathrm{p}<0.05$ and $\mathrm{p}<0.01$ respectively.

BF; before disinfection, AF; after disinfection, GPC; Gram positive cocci mostly staphylococci, GPB; Gram positive bacilli mostly Bacillus and diphtheroides; GNB; Gram negative bacilli including Pseudomonas and the Enterobacteriaceae, *Molds were mostly Aspergillus, Penicillium and white molds, " presence of bacteria or fungi

\section{DISCUSSION}

The hand is like the crane that picks readily from one place and deposits in another. Also, it can be likened to the housefly that visits both refuse and household's spreading germs. Therefore, the role of the hand as an agent of mechanical transmitter of pathogens cannot be debated as this has been recognized for long as a bane in the 


\begin{tabular}{|c|c|c|c|c|c|}
\hline \multirow[t]{2}{*}{$\begin{array}{l}\text { Microorganisms } \\
\text { isolated }\end{array}$} & \multirow{2}{*}{$\begin{array}{c}\text { Toilet } \\
\text { Practice } \\
\text { Tissue paper } n \text {; } \\
228\end{array}$} & \multirow[b]{2}{*}{$\begin{array}{c}\text { Water n; } \\
174\end{array}$} & \multicolumn{2}{|c|}{$\begin{array}{c}\text { Finger Nails } \\
\text { Type }\end{array}$} & \multirow[b]{2}{*}{$\begin{array}{c}\text { Acrylic nail } \\
\text { n; } 20\end{array}$} \\
\hline & & & $\begin{array}{l}\text { Long n; } \\
107\end{array}$ & $\begin{array}{c}\text { Short n; } \\
275\end{array}$ & \\
\hline $\begin{array}{l}\text { Pseudomonas } \\
\text { aeruginosa }\end{array}$ & $35(15.4)^{a}$ & $28(16.1)^{a}$ & $\begin{array}{c}42 \\
(39.3)^{x}\end{array}$ & $12(4.4)^{y}$ & $9(45)^{x}$ \\
\hline Escherichia coli & $70(30.7)^{a}$ & $40(23.0)^{b}$ & $\begin{array}{c}60 \\
(56.1)^{x}\end{array}$ & $\begin{array}{c}38 \\
(13.8)^{y}\end{array}$ & $12(60)^{x}$ \\
\hline Klebsiella spp & $78(34.2)^{a}$ & $55(31.6)^{a}$ & $\begin{array}{c}83 \\
(77.6)^{x}\end{array}$ & $\begin{array}{c}34 \\
(12.4)^{y}\end{array}$ & $16(80)^{x}$ \\
\hline Enterobacter spp. & $52(22.8)^{a}$ & $27(15.5)^{b}$ & $\begin{array}{c}44 \\
(41.1)^{x}\end{array}$ & $20(7.3)^{y}$ & $15(75)^{z}$ \\
\hline Proteus spp. & $67(29.4)^{a}$ & $10(5.8)^{b}$ & $\begin{array}{c}41 \\
(38.3)^{x}\end{array}$ & $\begin{array}{c}29 \\
(10.6)^{y}\end{array}$ & $7(35)^{x}$ \\
\hline $\begin{array}{l}\text { Enterococcus } \\
\text { faecalis }\end{array}$ & $55(24.2)^{a}$ & $30(17.4)^{a}$ & $\begin{array}{c}58 \\
(54.2)^{x}\end{array}$ & $15(5.5)^{y}$ & $12(60)^{x}$ \\
\hline Staphylococcus spp. & $118(51.8)^{a}$ & $84(48.3)^{a}$ & $\begin{array}{c}99 \\
(92.5)^{x}\end{array}$ & $\begin{array}{c}92 \\
(33.5)^{y}\end{array}$ & $11(55)^{z}$ \\
\hline Streptococcus spp. & $36(15.8)^{a}$ & $25(14.4)^{a}$ & $\begin{array}{c}38 \\
(35.5)^{x}\end{array}$ & $18(6.6)^{y}$ & $5(25)^{z}$ \\
\hline Bacillus spp. & $37(16.2)^{a}$ & $17(9.8)^{b}$ & $\begin{array}{c}26 \\
(24.3)^{x}\end{array}$ & $19(6.9)^{y}$ & $19(95)^{z}$ \\
\hline Candida albicans & $114(50.0)^{a}$ & $\begin{array}{c}155 \\
(89.1)^{b}\end{array}$ & $\begin{array}{c}95 \\
(88.8)^{x}\end{array}$ & $\begin{array}{c}162 \\
(58.9)^{y}\end{array}$ & $12(60)^{y}$ \\
\hline
\end{tabular}

${ }^{1}$ Values in parenthesis are in percentages, n; number of subjects sampled. Subjects under this category received the same treatment of hand washing with soap, followed with the application of sanitizer before samples were collected; superscripts along the same row with different letters indicate significance at $\mathrm{p}<0.05$.

Table 6 Microorganisms isolated from door handles and hand contacts

\begin{tabular}{ccccccccccc}
\hline $\begin{array}{c}\text { Organ- } \\
\text { isms }\end{array}$ & $\begin{array}{c}\text { Frequency of isolation (\%) from } \\
\text { door handles and contacts hands } \\
\text { classroom }\end{array}$ & & & & & & & & & \\
laboratorie: & toilets \\
Can- & BF & offices & BF & AF & BF & AF & BF & AF \\
dida & $5(25)$ & 0 & 8 & 0 & 12 & 2 & 15 & 4 \\
Moulds & $20(100)$ & $(0)$ & $(40)$ & $(0)$ & $(60)$ & $(10)$ & $(75)$ & $(20)$ \\
& & 0 & 20 & 0 & 20 & 4 & 20 & 4 \\
GPC & $18(90)$ & $(0)$ & $(100)$ & $(0)$ & $(100)$ & $(20)$ & $(100)$ & $(20)$ \\
& & 0 & 18 & 0 & 20 & 2 & 20 & 6 \\
GPB & $12(60)$ & $(0)$ & $(90)$ & $(0)$ & $(100)$ & $(10)$ & $(100)$ & $(30)$ \\
& & 6 & 10 & 3 & 17 & 5 & 15 & 2 \\
GNB & $8(40)$ & $(30)$ & $(50)$ & $(15)$ & $(85)$ & $(25)$ & $(75)$ & $(10)$ \\
& & 3 & 6 & 0 & 15 & 5 & 18 & 4 \\
Hand & $18(90)$ & $(15)$ & $(30)$ & $(0)$ & $(75)$ & $(25)$ & $(90)$ & $(20)$ \\
con- & & 2 & 15 & 0 & 20 & 3 & 18 & 2 \\
tacts* & & $(10)$ & $(75)$ & $(0)$ & $(100)$ & $(15)$ & $(90)$ & $(10)$ \\
\hline
\end{tabular}


control of infectious diseases Ayliffe et al. (1988); "Essential Practice for Infection Prevention and Control: Guidance for Nursing Staff" (2014); Knittle et al. (1975); "Report on the Burden of Endemic Healthcare-associated Infection Worldwide: Clean Care is Healthy Care" (2011). The 2014 EBOLA epidemics in Africa were associated with the touch phenomenon that required that suspect cases be isolated. Instructively, with the hands we render help, greet, feed, work and ironically transmit disease or kill. The present study became necessary and was inspired by the EBOLA epidemic that resulted in installation in the entrances of major buildings in our University sanitizer dispensers for regular disinfection of the hand. Consequent to which this study was carried out to investigate various habits that may aggravate spread of germs by hands and how hand washing can be effective in reducing microbial burden of the hand. Similarly, this study also answered the question is sanitizers effective without hand washing?

The results of this study showed that hand washing is only effective when it is regular and properly done and the minimal requirement is that hands should be washed with water and soap. The application of sanitizers will provide additional safety measures, especially if hands have come in contact with soiled materials. The results from unwashed hands showed build-up of microorganisms both bacteria and fungi far in excess of washed and sanitized hands. Particularly of concern is the presence of enteric bacteria and candida in hands. Although hand hygiene compliance in the general population has not been extensively studied, Judah et al. Judah et al. (2009) documented that one-quarter of adults in the general population show microorganisms of fecal origin on their hands, which implies low hand hygiene compliance after using the toilet. Though in this study, the subjects were briefed on the 5 Moments of hand washing and washed a video clip, it was also observed that many find it difficult to go through the procedure accurately as instructed. This may account for the persistence of microorganisms even after hand washing and the application of sanitizer as seen in this study and reported by other investigators Curtis and Cairncross (2003); Tambekar et al. (2007). The present study demonstrated a reduction in microbial population by 5-9 $\log _{10}$ CFU when sanitizer was used after hand washing and by 2-7 $\log _{10}$ CFU when sanitizer was applied to dry hand. This encouraging finding with the use of sanitizer alone will be a remedy in situations where there is shortage of water Lauharanta et al. (1991); Winnefeld et al. (2000). Consequently, it will be a good practice to carry a small jar of sanitizer in our hangs-on. The National Patient Safety Agency NPSA (2008) recommends that hand gel must be available at the 'point of care' in all primary and secondary care setting; this is in view to curb disease transmission. The absence of sanitizers and sanitary towels in the hospitals studied shortly after the EBOLA scourge was over is a clear indictment on the management of these healthcare Centers and also apathy on the part of regulatory body that should enforce compliance. With the food vending outlets these sanitary requirements were met because the regulatory agencies for food and drug regulation shut defaulting operators' premises. 
Hand hygiene is acknowledged as the single most important measure to prevent nosocomial infections in the healthcare setting. Similarly, in non-clinical settings, hand hygiene is recognized as a key element in helping prevent the spread of infectious diseases Babeluk et al. (2014). Pittet et al. Pittet et al. (1999) reported that hands of healthcare workers (HCW) become heavily contaminated during contactactivities with patients involving wound dressing, intravascular catheter care, respiratory tract care and the handling of patient secretions. The result of the present study that used visits to the toilet to pass out excretal simulated soiled conditions as in handling patients' secretions and showed consistently build-up in the hand microflora mostly with Gram-negative bacteria. This therefore implied that in addition to influencing the degree of contamination, objects touched or activities engaged in may determine the type of microorganisms colonizing the hands. This equally accounts for the heterogeneity of microorganisms isolated from the hands of the subjects. For example, Strausbaugh et al. Strausbaugh et al. (1994) reported high carriage of yeasts in hands of hospital personnel in addition to Gram positive bacteria.

This present study calls for the need for regular education on hand washing hygiene. It is common practice for people to rinse their hands after using the toilet. This is not hand washing. Rinsing or wetting the hand is shown by this study to increase the microbial load, just as perspiring hands contain more readily transmissible microorganisms "Guidance on the Management of Waste arising from Health, Social and Personal Care" (2011); "Royal College of Nursing. Infection Prevention and Control Minimum Standards, London: RCN. Publication code: 002 725” (2009). Wet surface contaminated is a more effective means of transmitting pathogens than dry surface. Similarly, this study showed that keeping of long nails reduces the effectiveness of hand washing as in all cases including the use of sanitizers, the fingertips of people with long nails harbor more and diversity of microorganisms. Therefore, addressing the question of habit and lifestyle first will enhance the effectiveness of hand hygiene.

\section{CONCLUSION}

The significance of the hand in disease transmission was played out in the Covid-19 pandemic that brought socio-economic activities to almost a standstill. In this scenario, the hand was the first stop point in considering prevention measures. Hands must be washed as frequent as it makes contact with surfaces, handshakes must be avoided and all who made hand contacts with confirmed cases or persons from high risk countries were subjected to the screening test. It can be said that hand and hygiene goes together. The question is how can good hygiene be practiced if there is no water. In the Millennium Development Goals (MDGs) which translated into the Sustainable Development Goals (SDGs), availability of water (clean drinking water) for all was a must requirement. Many nations are yet to achieve MDGs so sustainability is far from it. It should be further emphasized that the campaign against Covid-19 
requires that hands be washed with soap and under running water for 20 seconds. The implicit implication being that homes and public places should have taps from which water runs all the time. This is an area many nations should consider now and post Covid-19. Principally also is the role played by hand sanitizers during this pandemic. It is therefore a necessity that hand sanitizer should become part of our personal and daily hangs on as a routine.

We have experienced many disease outbreaks where hygiene practices were the first intervention. But hygiene should be a normal lifestyle and not a response to crises as has been the case during disease outbreaks. Therefore, the results of this study which set out to determine various socio-economic and psychological factors that may impinge on the hygiene consciousness of the people can be said to have been achieved. Behavioral factor was principally responsible for non-compliance with laid down hygiene requirements. From the outset of this study the question asked was how frequent should hands be washed. The Covid-19 pandemic and the outcome of this study have provided answer to that question. Hands should be washed as frequent as you want to stay safe.

The sequence of events that will lead to hand transmission of pathogens as highlighted in a healthcare setting and which can be applicable elsewhere include:

- Organisms present on the patient's skin, or that has been shed onto inanimate objects in close proximity to the patient, must be transferred to the hands of HCWs.

- These organisms must then be capable of surviving for at least several minutes on the hands of personnel.

- Next, hand washing or hand antisepsis by the worker must be inadequate or omitted entirely, or the agent used for hand hygiene must be inappropriate.

- Finally, the contaminated hands of the caregiver must come in direct contact with another patient, or with an inanimate object that will come into direct contact with the patient.

In extrapolating the above to community spread of diseases, the following should be considered in devising preventing measures:

- The handles of doors and flush systems how frequent are these disinfected?

- Are there adequate conveniences and washrooms in public places?

- Is there adequate and constant supply of water?

- Are there educational materials in public places that say for example, wash hands and use the sanitizer?

Finally, this study has shown the significance of the hand as an agent of disease transmission and discussed measures that can help reduce incidences of diseases associated with hand contact. Though this study had shown that a combination of hand washing and the use of a sanitizer will keep microorganisms away from the hand, the 
question that needs to be asked is how frequent do we carry out this process especially outside the hospital setting. The panacea will be after any activity that involved soiling the hands with body fluid, animals' waste or products or when we have a feeling that our hands may be dirty.

\section{REFERENCES}

Ayliffe, G. A. J., Babb, J. R., Davies, J. G., \& Lilly, H. A. (1988). Hand disinfection: a comparison of various agents in laboratory and ward studies. Journal of Hospital Infection, 11(3), 226-243. Retrieved from https://dx.doi.org/10.1016/0195-6701(88)90101-6 10.1016/0195-6701(88)90101-6

Babeluk, R., Jutz, S., Mertlitz, S., Matiasek, J., \& Klaus, C. (2014). Hand Hygiene - Evaluation of Three Disinfectant Hand Sanitizers in a Community Setting. PLoS ONE, 9(11), e111969-e111969. Retrieved from https://dx.doi.org/10.1371/journal.pone 0111969 10.1371/journal.pone.0111969

British Association for Parenteral and Enteral Nutrition. (2008). Combating Malnutrition: Recommendations for Action. Report from the Advisory Group on Malnutrition. In BAPEN, R. BAPEN, E. M, \& R. CA (Eds.), .

The Cost of Disease Related Malnutrition in the UK and Economic Considerations for the use of Oral Nutritional Supplements (ONS) in Adults. (2005).

Currie, L., Lecko, C., Gallagher, R., \& Sunley, K. (2011). Safety: Principle of Nursing Practice C. Nursing Standard., 25, 35-37.

Curtis, V., \& Cairncross, S. (2003). Effect of washing hands with soap on diarrhoea risk in the community: a systematic review. The Lancet Infectious Diseases, 3(5), 275-281. Retrieved from https://dx.doi.org/10.1016/s1473-3099(03)00606-6 10.1016/s1473 -3099(03)00606-6

Department, \& Health. (2009). The Health and Social Care Act 2008: Code of Practice for Health and Adult Social Care on the Prevention and Control of Infections and Related Guidance.

Essential Practice for Infection Prevention and Control: Guidance for Nursing Staff. (2014). Royal College of Nursing.

Francesconi, P., Yoti, Z., Declich, S., Onek, P. A., Fabiani, M., Olango, J., Andraghetti, R., Rollin, P. E., Opira, C., Greco, D., \& Salmaso, S. (2003). Ebola Hemorrhagic Fever Transmission and Risk Factors of Contacts, Uganda1. Emerging Infectious Diseases, 9(11), 1430-1437. Retrieved from https://dx.doi.org/10.3201/eid0911.030339 10.3201/ eid0911.030339

Guidance on the Management of Waste arising from Health, Social and Personal Care. (2011). London: RCN. Publication.

Judah, G., Aunger, R., Schmidt, W.-P., Michie, S., Granger, S., \& Curtis, V. (2009). Experimental Pretesting of Hand-Washing Interventions in a Natural Setting. American Journal of Public Health, 99(S2), S405-S411. Retrieved from https://dx.doi.org/10.2105/ajph .2009.164160 10.2105/ajph.2009.164160

Knittle, M. A., Eitzman, D. V., \& Baer, H. (1975). Role of hand contamination of personnel in the epidemiology of gram-negative nosocomial infections. The Journal of Pediatrics, 86(3), 433-437. Retrieved from https://dx.doi.org/10.1016/s0022-3476(75)80980-2 10.1016/s0022-3476(75)80980-2

Lauharanta, J., Ojajärvi, J., Sarna, S., \& Mäkelä, P. (1991). Prevention of dryness and eczema 
of the hands of hospital staff by emulsion cleansing instead of washing with soap. Journal of Hospital Infection, 17(3), 207-215. Retrieved from https://dx.doi.org/10.1016/ 0195-6701(91)90232-w 10.1016/0195-6701(91)90232-w

NPSA. (2008). National Patient Safety Agency. Clean Hands Save Lives: Patient Safety Alert, London. Retrieved from www.nrls.npsa.nhs.uk/resources

Nutrition Screening Survey in the UK in 2007: A Report by BAPEN. Nutrition Screen Survey and Audit of Adults on Admission to Hospital, Care Homes and Mental Health Units. (2008).

Pittet, D., Dharan, S., Touveneau, S., Sauvan, V., \& Perneger, T. V. (1999). Bacterial Contamination of the Hands of Hospital Staff During Routine Patient Care. Archives of Internal Medicine, 159(8), 821-821. Retrieved from https://dx.doi.org/10.1001/archinte.159 .8 .821 10.1001/archinte.159.8.821

Pratt, R. J., Pellowe, C. M., Wilson, J. A., Loveday, H. P., Harper, P. J., Jones, S. R. L. J., McDougall, C., \& Wilcox, M. H. (2007). epic2: National Evidence-Based Guidelines for Preventing Healthcare-Associated Infections in NHS Hospitals in England. Journal of Hospital Infection, 65, S1-S59. Retrieved from https://dx.doi.org/10.1016/s0195-6701(07)60002-4 10.1016/s0195-6701(07)60002-4

Report on the Burden of Endemic Healthcare-associated Infection Worldwide: Clean Care is Healthy Care. (2011)

Royal College of Nursing. Infection Prevention and Control Minimum Standards, London: RCN. Publication code: 002 725. (2009).

Strausbaugh, L. J., Sewell, D. L., Ward, T. T., Pfaller, M. A., Heitzman, T., \& Tjoelker, R. (1994). High frequency of yeast carriage on hands of hospital personnel. Journal of Clinical Microbiology, 32(9), 2299-2300. Retrieved from https://dx.doi.org/10.1128/jcm.32.9 .2299-2300.1994 10.1128/jcm.32.9.2299-2300.1994

Tambekar, D. H., Shirsat, S. D., Suradkar, S. B., Rajankar, P. N., \& Banginwar, Y. S. (2007). Prevention of Transmission of Infectious Disease: Studies on Hand Hygiene in Health-care Among Students. Continental Journal Biomedical Sciences, 1, 6-10.

Winnefeld, M., Richard, M. A., Drancourt, M., \& Grob, J. J. (2000). Skin tolerance and effectiveness of two hand decontamination procedures in everyday hospital use. British Journal of Dermatology, 143(3), 546-550. Retrieved from https://dx.doi.org/10.1111/ j.1365-2133.2000.03708.x 10.1111/j.1365-2133.2000.03708.x

World Health Organization. Ebola Haemorrhagic Fever in Sudan. (1976). Bulletin of World Health Organization, 56, 247-270.

World Health Organization. Ebola Haemorrhagic Fever in Zaire. (1976). Bulletin of World Health Organization, 56, 271-293. 